\title{
La crisis por COVID-19 hará necesario el uso de cánulas de alto flujo, además de ventilladores mecánicos
}

\section{High-flow cannulas will be required with current COVID-19 crisis, not only mechanical ventilators}

\author{
Edgar Santos, ${ }^{1 *}$ Francisco L. Ramírez-Cuapio, ${ }^{1}$ Lorena T. Remes-Díaz ${ }^{2}$ y Renán Sánchez-Porras ${ }^{1}$ \\ ${ }^{1}$ University of Heidelberg, Department of Neurosurgery, Heidelberg, Alemania; ${ }^{2}$ Instituto Mexicano del Seguro Social, Hospital General Regional \\ 46, Guadalajara, Jalisco, México
}

COVID-19 se caracteriza por una progresión del síndrome de dificultad respiratoria aguda, ${ }^{1}$ que va de grado leve a severo. ${ }^{2}$ Un porcentaje de los pacientes graves requerirá intubación endotraqueal y ventilación mecánica, por ello, ingenieros mexicanos de diferentes lugares han tenido la iniciativa de crear ventiladores mecánicos. ${ }^{3-5}$

Si bien el ventilador artificial es uno de los últimos recursos para los pacientes más graves, existen pacientes que se encuentran en el límite de no cumplir los criterios para la intubación; si se intuban directamente, se estaría dejando pasar la oportunidad de que superen la fase grave con oxígeno suplementario de alta concentración. La intubación per se induce daño pulmonar, aumenta el riesgo de sobreinfección y los días de estancia en terapia intensiva. ${ }^{6,7}$ Asimismo, el uso no selectivo de ventiladores artificiales disminuye la oportunidad de los pacientes que requieren el ventilador para sobrevivir e incrementa los costos de atención institucional, tanto de recursos humanos como materiales.

Las cánulas de oxígeno de alto flujo tienen la función de proveer altas concentraciones de oxígeno al paciente, las cuales no se alcanzan con las puntas nasales o máscaras normales..$^{8-10}$ En algún momento, el oxígeno suplementario con puntas nasales no es tolerado por el paciente y produce resequedad, pérdida de calor en las vías respiratorias, sangrados, lesiones de la mucosa nasal y dolor. Las cánulas de oxígeno de alto flujo son mucho más simples que un ventilador artificial, pues su única función es hacer una mezcla de oxígeno con agua caliente para humectar, poner la mezcla de gases a temperatura corporal y disminuir la incomodidad de tener oxígeno a altas concentraciones en las vías aéreas (Figura 1). Además del beneficio de no necesitar sedación, disminuyen el estrés, mejoran la respiración y promueven respiraciones profundas, incrementando la ventilación alveolar. Adicionalmente, el paciente requiere menos cuidados por parte del personal de enfermería, incluso puede comer, beber y dormir en condiciones normales. Debido a la naturaleza de los pacientes con COVID-19, cuyo principal problema es la hipoxia, la cánula de oxígeno de alto flujo parece más conveniente que la ventilación intermitente no invasiva dado que las necesidades respiratorias pueden superar los $50 \mathrm{~L} /$ hora y una máscara normal solo puede aportar aproximadamente $10 \mathrm{~L} /$ hora de oxígeno, además de mezclar el aire con el oxígeno; con las cánulas de alto flujo se puede proporcionar hasta 50 litros de oxígeno, sin la necesidad de diluir con aire. ${ }^{8}$

Además de las ventajas anteriores es importante hacer dos observaciones:

- El uso de cánulas de oxígeno de alto flujo puede producir aerosol, por lo cual el procedimiento debe llevarse a cabo en cuartos con presión negativa. Cuando no sea posible implementar presión negativa, puede emplearse artefactos para aislar los aerosoles del paciente, por ejemplo, coberturas de diferentes materiales, cascos, etcétera.

- Este recurso material no se encuentra disponible en las instituciones públicas de segundo nivel que han sido designadas como centros de reconversión COVID y el que existe en los hospitales de tercer nivel probablemente será insuficiente.

El sistema de oxígeno de alto flujo reduciría la necesidad de intubación en pacientes agudos ${ }^{11,12}$ y tiene el
Correspondencia:

*Edgar Santos

E-mail: dredgarsantos@hotmail.com
Gac Med Mex. 2020;156:259-260

Disponible en PubMed

www.gacetamedicademexico.com

0016-3813/@ 2020 Academia Nacional de Medicina de México, A.C. Publicado por Permanyer. Este es un artículo open access bajo la licencia CC BY-NC-ND (http://creativecommons.org/licenses/by-nc-nd/4.0/). 


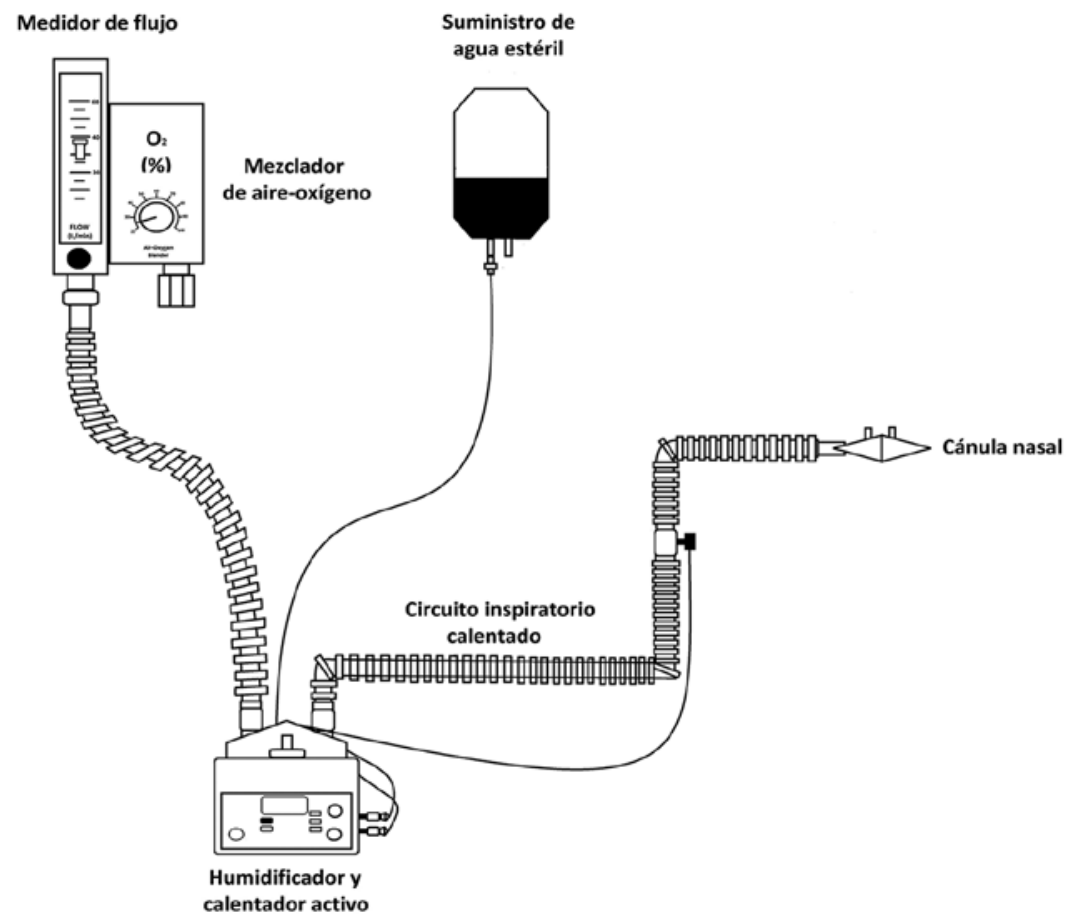

Figura 1. Esquema general de un modelo de cánula de alto flujo.

potencial de salvar vidas debido a la facilidad de su uso en la práctica clínica, la reducción de costos, la limitación del riesgo biológico del equipo médico durante la protección de la vía aérea y la liberación de ventiladores para otros pacientes que sí los requieran. A su vez, puede disminuir los días de estancia en la unidad de cuidados intensivos, lo cual será especialmente importante cuando los ventiladores y las camas en terapia intensiva sean escasos o no estén disponibles por la cantidad de pacientes.

Sugerimos a las autoridades de gobierno la compra urgente de estos dispositivos y a los ingenieros, empresas y centros de investigación, enfocarse también en la producción de cánulas de alto flujo.

\section{Conflicto de intereses}

Los autores no tienen conflicto de intereses.

\section{Financiamiento}

Los autores no recibieron patrocinio para llevar a cabo este escrito.

\section{Bibliografía}

1. Palacios-Cruz M, Santos E, Velázquez-Cervantes MA, León-Juárez M. COVID-19, una emergencia de salud pública mundial. Rev Clin Esp. 2020. [Epub ahead of print]

2. Huang C, Wang Y, Li X, Ren L, Zhao J, Hu Y, et al. Clinical features of patients infected with 2019 novel coronavirus in Wuhan, China. Lancet. 2020;395:497-506.

3. Cano LC. Ingenieros crean respirador artificial básico de bajo precio. El Diario [en línea] 2020 Abr 03.

4. Roa P. Ingenieros mexicanos crean respiradores sin fines de lucro para pacientes con coronavirus. Infobae [en línea]. 2020 Mar 31.

5. Barrera KH. Profesor de la Universidad de Sonora crea respirador artificial de bajo costo. SDPnoticias [en línea] 2020 Mar 31.

6. Girou E, Schortgen F, Delclaux C, Brun-Buisson C, Blot F, Lefort Y, et al. Association of noninvasive ventilation with nosocomial infections and survival in critically ill patients. JAMA. 2000;284:2361-2367.

7. Girou E, Brun-Buisson C, Taillé S, Lemaire F, Brochard L. Secular trends in nosocomial infections and mortality associated with noninvasive ventilation in patients with exacerbation of COPD and pulmonary edema. JAMA. 2003;290:2985-2991.

8. Roca O, Riera J, Torres F, Masclans JR. High-flow oxygen therapy in acute respiratory failure. Respir Care. 2010;55:408-413.

9. Lenglet H, Sztrymf B, Leroy C, Brun P, Dreyfuss D, Ricard JD. Humidified high flow nasal oxygen during respiratory failure in the emergency department: feasibility and efficacy. Respir Care. 2012:57:1873-1878.

10. Stéphan F, Barrucand B, Petit P, Rézaiguia-Delclaux S, Médard A, Delannoy $B$, et al. High-flow nasal oxygen vs noninvasive positive airway pressure in hypoxemic patients after cardiothoracic surgery: a randomized clinical trial. JAMA. 2015:313:2331-2339.

11. Frat JP, Thille AW, Mercat A, Girault C, Ragot S, Perbet S, et al. High-flow oxygen through nasal cannula in acute hypoxemic respiratory failure. N Engl J Med. 2015;372:2185-2196.

12. Alhazzani W, Møller MH, Arabi YM, Loeb M, Gong MN, Fan E, et al. Surviving sepsis campaign: guidelines on the management of critically ill adults with coronavirus disease 2019 (COVID-19). Intensive Care Med. 2020:1-34. 